\title{
Repair of antero lateral ligament injuries in acute anterior cruciate ligament tears: an in vivo study using navigation
}

\author{
E. Monaco, A. Ponzo, D. Lupariello, P. Rota, M. Fabbri, \\ R. Lanzetti, D. Mazza, A. Ferretti \\ "La Sapienza" University Of Rome, Sant'Andrea Hospital, Traumatology \\ Sports Center "Kilk Kilgour"
}

\section{CORRESPONDING AUTHOR:}

\section{Antonio Ponzo}

"La Sapienza" University, Sant'Andrea

hospital, Traumatology Sports

Center "Kilk Kilgour"

Via di Grottarossa 1035

E-mail: antonio.ponzo@hotmail.it

Phone: 00393483639510

Phone: 00390633775818

Fax: 00390633775887

DOI:

10.32098/mltj.02.2019.07

LEVEL OF EVIDENCE:

4

\begin{abstract}
SUMMARY
Background. To evaluate the effect of antero-lateral ligament (ALL) repair on the anterior-tibial translation (ATT) and the axial-tibial rotation (ATR) during the pivot-shift test with a navigation system in patient undergoing acute anterior cruciate ligament (ACL) reconstruction of acute injuries of anterolateral compartment in cases of acute ACL tears. Methods. Ten consecutive patients operated for an acute ACL reconstruction were enrolled in this prospective study. The pivot-shift test was repeated after all three steps of the procedure with navigation system: preoperatively, after repair of the ALL and after ACL reconstruction. This system provided a real-time calculation of ATT and ATR during the pivot shift test. Results. The mean ATT during the dynamic pivot-shift test was $15 \mathrm{~mm} \pm 3 \mathrm{~mm}$ before ACL reconstruction and ALL repair, $11 \mathrm{~mm} \pm 2 \mathrm{~mm}$ after ALL repair and $6 \mathrm{~mm} \pm 3 \mathrm{~mm}$ after ALL repair and ACL reconstruction. The mean ATR during the dynamic pivot-shift test was $22^{\circ} \pm 12^{\circ}$ before ACL reconstruction and ALL repair, $16^{\circ} \pm 3^{\circ}$ after ALL repair and $10^{\circ} \pm 4^{\circ}$ after ALL repair and ACL reconstruction. Conclusion. The repair of the antero-lateral compartment had a statistically significant effect on ATR, with no effect on ATT. The addition of the ACL reconstruction produced a significant effect on ATT, but no effect on ATR.
\end{abstract}

\section{KEY WORDS}

ACL; Antero-lateral ligament; navigation; pivot-shift

\section{BACKGROUND}

Anterior Cruciate Ligament (ACL) reconstructions have specifically focused on ACL restore, providing good to excellent results in most cases. However, a persistent instability of the knee at follow-up, especially under rotatory load as revealed by a positive pivot-shift test (1), has been reported in $11 \%$ to $30 \%$ patients by many authors $(2,3)$. As a matter of fact, the persistence of a certain degree of pivot-shift is related to poor functional outcomes (4). The pivot-shift test is a complex multi-planar phenomenon that involves rotation and translation of the tibia with the knee subjected to valgus stress and internal rotation ranging from full extension to 30-40 degrees of flexion. However, its major limitation is that, as it is manually performed by the clinician, it is strongly correlated to the feelings of the examiner, with an obvious interobserver variability (5-6).
Some authors have focused on the ligaments of the lateral aspect of the knee acting as secondary restraints of the ACL deficient knee, whose deficiency could result in the only fair control of pivot-shift phenomenon after ACL reconstruction (ACLr). Recent studies have further investigated the anatomy of the so called "antero-lateral ligament" (ALL) (7-9), that has been clearly identified as a distinct ligamentous structure in 83 to $100 \%$ of knees. Moreover, the role of the anterolateral structures in rotational control of the knee has been demonstrated recently by several robotic cadaveric studies where the effect of a lesion of the ALL-in addition to an ACL injury was evaluated (10). Despite this, the precise role of the ALL in eliminating the pivot shift remains controversial (11). This disparity exists secondary to the nature of cadaveric research and the subsequent creation of artificial injury patterns that are not a true representation of in-vivo characteristics. 
In a recent study, surgical exploration of the lateral compartment was performed on sixty consecutive patients undergoing acute ACL reconstruction. The authors reported a prevalence of lesions of the anterolateral complex of about $90 \%$, with about $20 \%$ of cases involving only the ALL, and their repair was suggested in order to improve knee stability (12).

The goal of the present study is to evaluate the role that anterolateral structures plays in anterolateral rotatory instability, as demonstrated by the pivot-shift sign detected by a navigation system during ACL surgery.

In particular the purpose of this study was to evaluate the effect of the repair of ALL with the aid of a navigation system with dedicated software to calculate anterior-tibial translation (ATT) and axial-tibial rotation (ATR) during the pivotshift test performed during ACL surgery (13); the hypothesis of this study was that ALL repair would reduce pivot-shift phenomenon in association with an intra-articular ACLr.

\section{MATERIALS AND METHODS}

\section{Patients selection}

A careful inspection of the anterolateral compartment in patients undergoing primary ACLr for an acute ACL tear (within 7 days from injury) was performed in a series of 60 consecutive patients in order to investigate prevalence and pattern of injuries of the anterolateral capsule and complex (12). Diagnosis of ACL lesion were performed using Lachman test and pivot-shift test. Varus and valgus stress testing were both negative at $0^{\circ}$ and $30^{\circ}$ of flexion. A $1.5 \mathrm{~T}$ MRI was also performed and confirmed the clinical diagnosis of ACL rupture, and demonstrated characteristic lateral femoral condyle bone bruising in 9/10 patients. Even though no ALL or anterolateral capsule lesion was reported by the radiologist, it was possible to identify abnormalities of the ALL according to previously reported imaging parameters of this structure (figure 1).

From the original 60 patients, 10 patients presenting a type III lesion (complete lesion involving only the ALL) met the inclusion criteria and were enrolled for the present study (figure 2). In all 60 patients, a lateral incision to access the antero-lateral capsule was made and a surgical repair of the lesion type was performed. Only patients presenting a type III lesion were considered for the present study in order to focus the investigation on ALL (12). Four out of the ten patients of this study presented a meniscal lesion treated with a partial meniscectomy: two patients presented a posterior horn lesion of medial meniscus, one patients presented a posterior horn lesion of lateral meniscus and one patient presented a middle body lesion of lateral meniscus. No patients presented ramp or root meniscal tears.

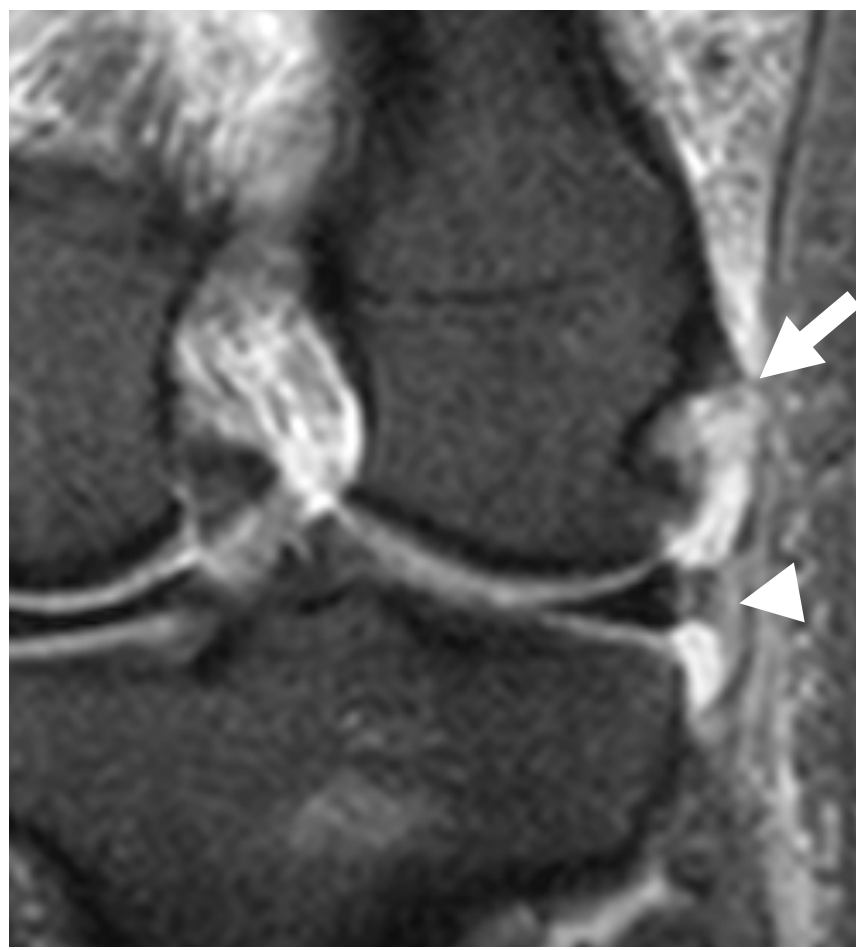

Figure 1. Antero-lateral ligament lesion on MRI. Coronal T2 weighted with fat saturation MRI images of a left knee with white arrows at the anterolateral ligaments and arrowheads at the inferior lateral genicular arteries. Left knee ACL was injured. Left knee ALL presented decreased thickness and increased signal. Proximal fibers were also irregular. Note that the inferior lateral genicular arteries are asymmetric.

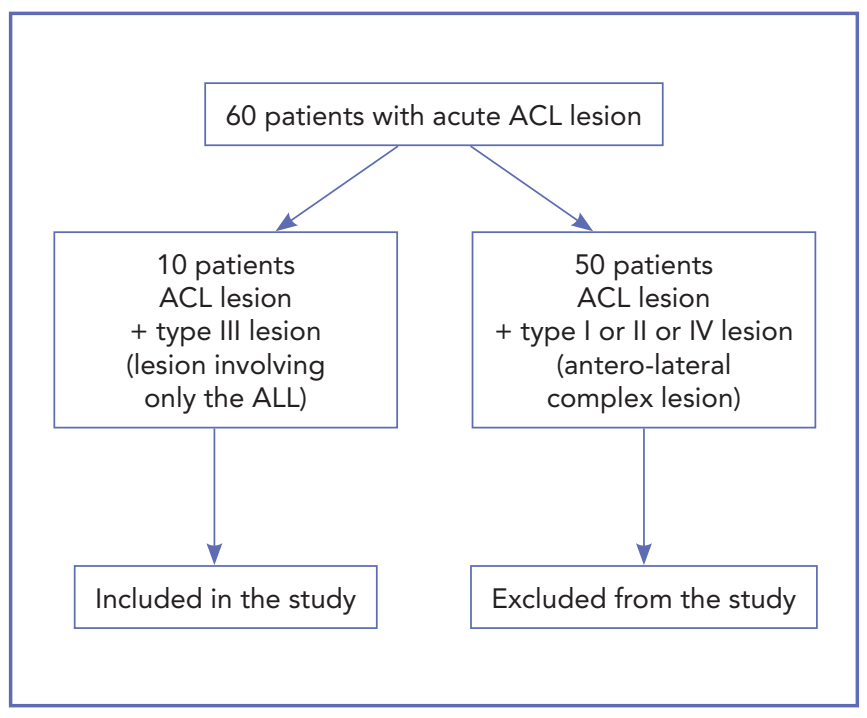

Figure 2. Flow chart of inclusion criteria. 
Exclusion criteria were: $\mathrm{BMI}>30$; age over 50 years; severe associated ligamentous injuries or laxity tests positive other than Lachman and pivot-shift test; cartilage damage (grade 3 or 4 according with Outerbridge classification), rheumatologic disorders or malalignment.

There were 7 males and 3 females with a mean age at surgery of 27 years (range 18-35 years). All patients had surgery using an anatomic single-bundle ACLr with doubled semitendinosus and gracilis tendons and a two incisions Out-in technique. A navigation system equipped with dedicated software (3.0 OrthoPilot ACL navigation system- B.Braun -Aesculap, Tuttlingen, Germany) was used for stability assessment. All the tests were performed by the senior surgeon.

All patients agreed to participate in the study and signed an informed consent form in accordance with the Declaration of Helsinki (1964).

The study meets the ethical standards of the journal (14).

\section{ALL repair}

Before surgery under general anesthesia all patients were clinically evaluated with Lachman and Pivot Shift test. Before proceeding with ACLr, in all patients the lateral compartment was approached by a "hockey stick incision"; the fascia lata was first inspected and then longitudinally divided along its fibers and elevated to expose lateral compartment from postero-lateral corner (PLC) to Gerdy's tubercle. The lateral compartment was carefully inspected and only patients presenting a type III lesion (isolated transverse lesion of the ALL, figure 3a) as classified by Ferretti et al. (12) were included in the study. The lesion was accurate-

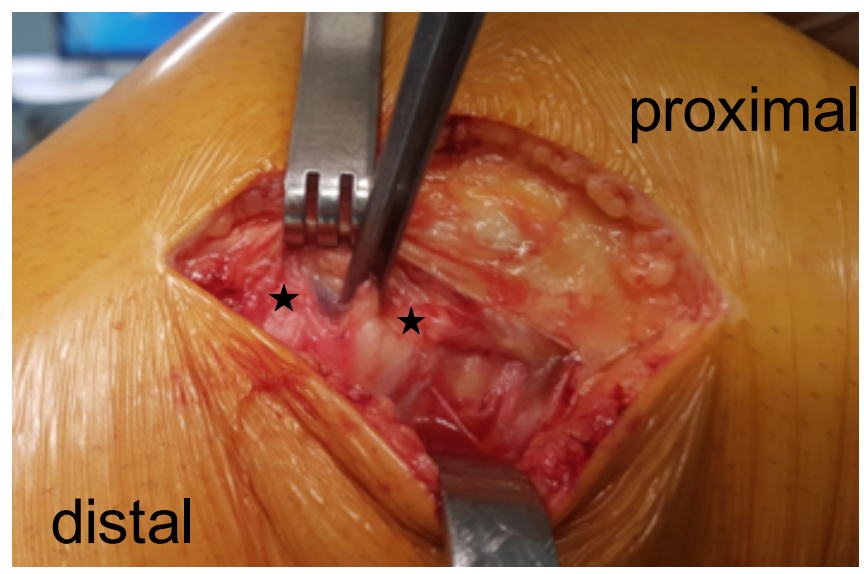

Figure 3a. Antero-lateral ligament lesion. Isolated lesion transverse lesion of the anterolateral compartment, type III as classified by Ferretti et al. (Left Knee). The black stars indicate the proximal and distal stamps of the ligament. ly repaired using absorbable parallel stitches with square knots (Coated VICRYL®, Ethicon, sized 3-0) in tension with the knee at 90 degrees of flexion and neutral rotation, with no any kind of complications (figure $3 \mathbf{b}$ ).

\section{Intra-articular Surgical Technique}

An arthroscopically assisted anatomic single bundle two-incision technique using doubled semitendinosus and gracilis tendons (DGST) autografts was performed. The patient is placed in a standard supine position with the tourniquet on the upper thigh. The knee is enabled to achieve at least $90^{\circ}$ of flexion. We standardly performed an out-in anatomic ACLr using a DGST graft. Tendons were harvested with an open tendon stripper. The free ends of the two tendons were prepared using a double-cross suture using absorbable stiches (Coated VICRYL®, Ethicon, Edinburgh, United Kingdom). The two tendons were doubled over the loop of the femoral fixation device (ACL TightRope®, Arthrex® , Naples, Florida, USA). Tibial tunnel was performed with the knee at $90^{\circ}$ of flexion using a standard guide at $60-65^{\circ}$. The guide was inserted through antero-medial (AM) portal and the guide wire is placed at the center of the tibial stump of the ACL. The diameter was chosen on the base of graft diameter. Femoral tunnel was performed with an out-in technique using a retro-drill device (FlipCutter ${ }^{\circledR}$, Arthrex ${ }^{\circledR}$, Naples, Florida, USA). The guide was inserted through the AM portal to achieve the anatomical femoral insertion of the native ACL between the resident ridge and the posterior wall of the lateral condyle. The half tunnel was performed with a retrograde drilling and the diameter was chosen on the basis of graft diameter. The graft was passed from tibial tunnel to

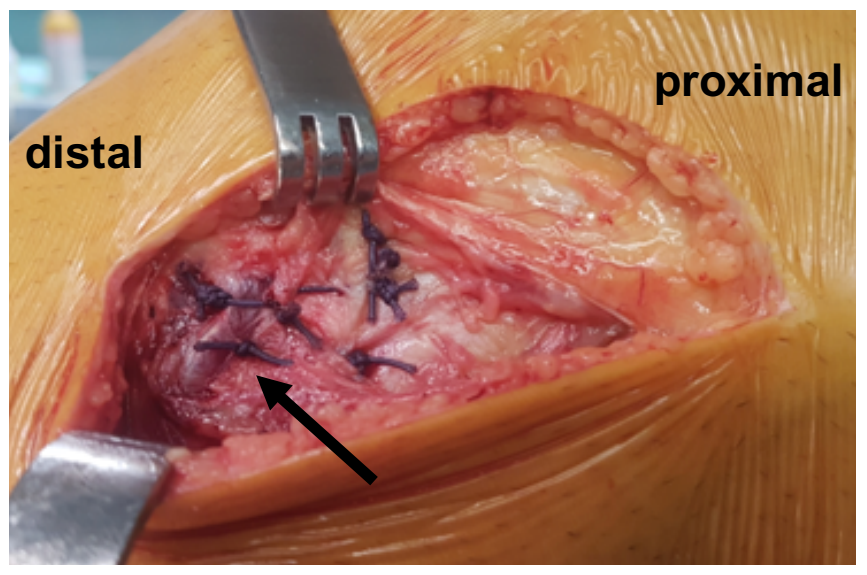

Figure 3b. Antero-lateral ligament repair. The type III lesion of anterolateral compartment was accurately repaired in tension using absorbable stitches (Left Knee). The black arrow indicates the ligament sutured.

Muscles, Ligaments and Tendons Journal 2019;9 (2) 
femoral tunnel using a passing wire with an out-in technique. The graft was fixed on the femur with ACL tight-rope. The passing suture was removed and the graft was progressively paced in the half tunnel by pulling alternatively the tensioning sutures. At this time, free ends of tendons were manually pulled from the tibial side and tensioned while 0 to $90^{\circ}$ of range of motion was applied to the knee. The graft was then fixed at the tibial side with the knee at $30^{\circ}$ of flexion using an absorbable interference screw (interference screw round delta, Arthrex ${ }^{\circledR}$, Naples, Florida, USA) with a diameter of 1 $\mathrm{mm}$ more that the tibial tunnel and a length of $35 \mathrm{~mm}$.

\section{Navigation}

The navigation system is a wireless system which does not require computed tomography (figure 4). Two $2.5 \mathrm{~mm}$ $\mathrm{K}$-wires were placed respectively in the distal portion of the femur and in the proximal portion of the tibia, and rigid bodies with reflective markers were attached. Extra-articular landmarks were registered by the system using an instrumented pointer. The extra-articular landmarks included the tibial tuberosity, the anterior edge of the tibia, and medial and lateral points of the tibial plateau. Kinematics of the knee joint were dynamically registered by the navigator during range of motion from full extension to $90^{\circ}$ of knee flexion. This system provided a real-time calculation and record of ATT and ATR at all degrees of knee flexion, while the surgeon manipulated the knee joint. According to the manufacturer, the error of the navigation system is estimated to be less than $1 \mathrm{~mm}$ or less than $1^{\circ}$.

The pivot-shift test was intra-operatively performed and measurements of ATT and ATR were recorded at three different steps: before reconstruction, after repair of the lateral compartment and lastly after ACLr.
The pivot-shift test was performed by the same surgeon 3 times at each step and the data was averaged by the navigation system. The test was performed using manual loads applied by the same surgeon to minimize inter-observer variability. ATT was expressed in $\mathrm{mm}$ and ATR in degrees. During the trial, a diagram showing the curve of ATT and ATR during the test was visualized and saved with a screenshot at the end of each step of the procedure.

\section{Statistical Analysis}

Statistical analysis was performed by statisticians of Regional Agency of Public Health. The values of the three variables were also recorded by a standard excel file (Microsoft Office, Microsoft Corporation, USA).

Power analysis was performed using alfa $=0.05$ and $\mathrm{N}$ (number of cases) $=10$ for each condition; the power was fixed at 0.8 .

Statistical analysis was performed for each variable (ATT, ATR) in the three-surgical step using two-way without replication analysis of variance (ANOVA). Both tests showed a statistical significant difference between averages of the three-surgical step, considering a significant level of 0.95. A paired Student t-test ( $\mathrm{p}$ value) was then performed to investigate for each variable (ATT, ATR) the statistical significant difference between their surgical steps.

Statistical analysis was performed using R (GNU GPL for Microsoft Windows; version 3.1.0).

\section{RESULTS}

At the pre-operative clinical evaluation, all patients had a positive Lachman test, 4 patients showed $2+$ and 6 patients showed $3+$ at pivot-shift test.

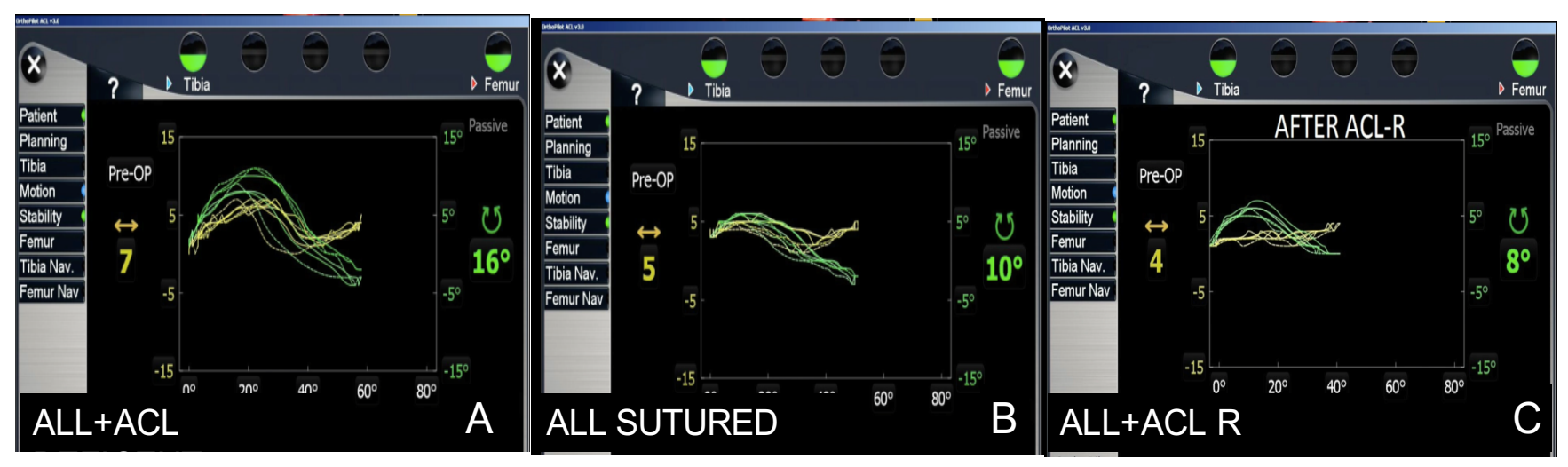

Figure 4. The navigation system.3.0 OrthoPilot software shows the screenshot of the three surgical steps recorded: $\mathrm{A}$, before $A L L$ repair and $A C L$ reconstruction; $B$, after ALL repair; $C$, after ALL repair and ACL reconstruction. 


\section{Navigator evaluation}

The mean ATT during the dynamic pivot-shift test was $15 \mathrm{~mm} \pm 3 \mathrm{~mm}$ before ACLr and anterolateral repair, $11 \mathrm{~mm} \pm 2 \mathrm{~mm}$ after anterolateral repair and $6 \mathrm{~mm} \pm 3 \mathrm{~mm}$ after ALL repair and ACLr.

The mean ATR during the dynamic pivot-shift test was $22^{\circ} \pm 12^{\circ}$ before ACLr and ALL repair, $16^{\circ} \pm 3^{\circ}$ after anterolateral repair and $10^{\circ} \pm 4^{\circ}$ after ALL repair and ACLr.

The repair of the lateral compartment had a statistically significant effect on ATR $(\mathrm{p}<0.001)$, with no effect on ATT $(\mathrm{p}=0.18)$. The addition of the ACLr produced a significant effect on ATT $(\mathrm{p}<0.05)$ but no effect on ATR $(\mathrm{p}=0.12)$.

After lateral repair, and before ACLr, the intra-operatively pivot-shift test, as manually evaluated by the surgeon, resulted as being negative for all patients.

Results are summarized in Table I and Table II.

\section{DISCUSSION}

The most important finding of this study is that direct repair of the ALL lesion in ACL deficient knee has a significant effect on ATR and no effect on ATT during the pivot-shift test, while subsequent ACLr has a significant effect on ATT and no effect on ATR. So, the primary hypothesis that repair of ALL in addition to ACL intraarticular reconstruction reduce the PS phenomenon is confirmed.

This study found that ALL was the primary restraint to tibial rotation, while ACL was the primary restraint to anterior tibial translation.

These findings seem to agree with other previous studies confirming that ACL and lateral structure function in a synergistic relationship to control pivot-shift phenomenon (15-17).

Another important finding of the present study relates to the identification of the anterolateral capsule and ligament

Table I. Anterior tibial translation and axial tibial rotation results

\begin{tabular}{llll}
\hline & $\begin{array}{l}\text { Before ALL and } \\
\text { ACL surgery }\end{array}$ & After ALL surgery & After ALL and ACL surgery \\
\hline $\begin{array}{l}\text { Anterior Tibial Translastion } \\
\text { (ATT) }\end{array}$ & $15 \pm 3 \mathrm{~mm}$ & $11 \pm 2 \mathrm{~mm}$ & $6 \pm 3 \mathrm{~mm}^{*}$ \\
\hline $\begin{array}{l}\text { Axial Tibial Rotation } \\
(\text { ATR })\end{array}$ & $22 \pm 12^{\circ}$ & $16 \pm 3^{\circ *}$ & $10 \pm 4^{\circ}$ \\
\hline
\end{tabular}

" $p<0.05$

The repair of the lateral compartment had a statistically significant effect on ATR ( $\mathrm{p}=0.0001)$, with no effect on ATT ( $\mathrm{p}=0.18)$. The addition of the ACL reconstruction produced a significant effect on ATT ( $\mathrm{p}=0.01)$ but no effect on ATR $(\mathrm{p}=0.12)$.

Table II. Anterior tibial translation and axial tibial rotation results

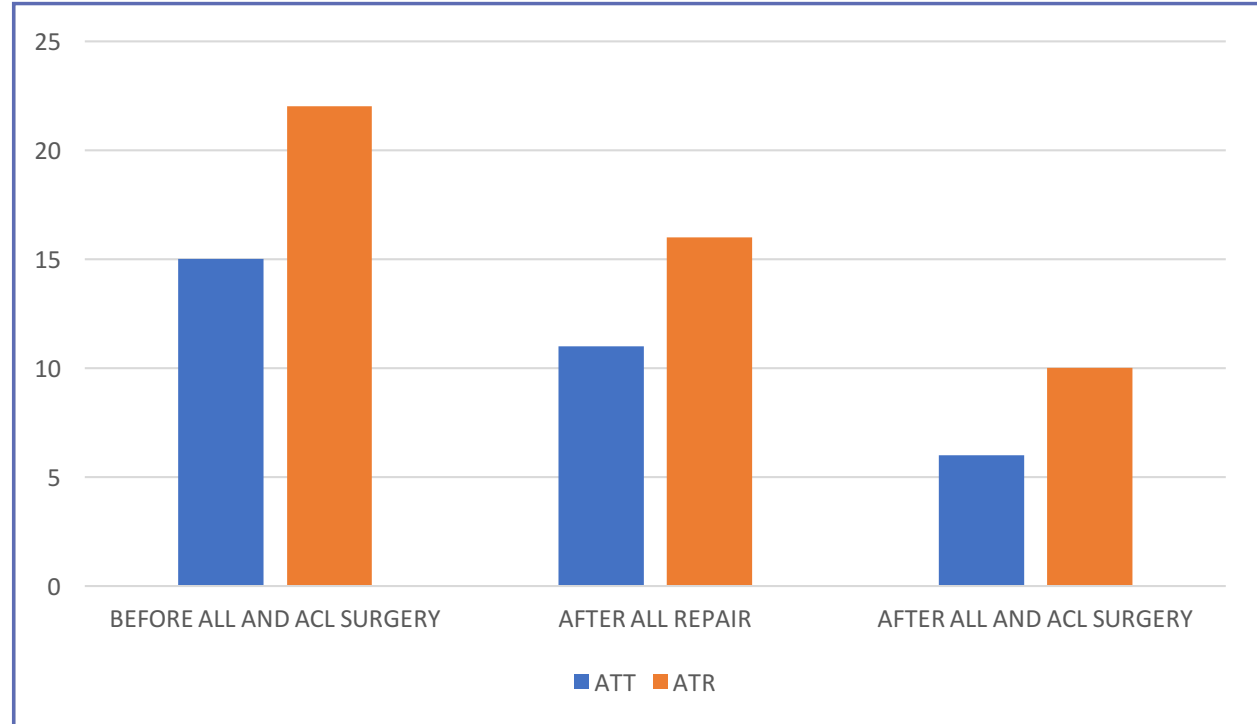

ATT (anterior tibial translation) is expressed in millimeters; ATR (axial tibial rotation) is expressed in grades. 
injury by MRI. This is perhaps not particularly surprising given that there appears to be considerable disparity between the rates of concomitant ALL injury identified in ACL deficient knees depending on whether the evaluation was surgical (12) or based on MRI $(18,19)$. This indicates that current imaging modalities are not sensitive enough and probably radiologists and knee surgeons are not yet familiar with this kind of lesions, thus explaining the injury assessment disparity in the current case report. This suggests that "normal" MRI should not preclude consideration of injury to the anterolateral structures of the knee.

Rotatory instability occurring during running in the ACL-deficient knee was first reported by Slocum and Larson and they also described a rotatory instability test, while the term "pivot-shift" was first used in 1976 (1). The pivot-shift phenomenon was well described by Bull and Ami (20) as a sudden rotation of the tibia relative to the femur for an ACL injured knee under a valgus torque at low angles of knee flexion. The two components are rotation component (rotation of the tibia about its long axis) and translational component (the anterior subluxation of the lateral tibial plateau followed by its sudden reduction under certain loading conditions). Both components affect the clinical featuring of pivot-shift phenomenon. Moreover, the rotational axis of the knee is located centrally near the spines in a normal condition while in the ACL deficient knee this axis of rotation moves toward the medial compartment which increases anterior tibial translation and internal rotation of the lateral compartment (21).

Based on the results of the present study, it is possible to speculate that ALL repair acting from the periphery became the primary restraint of the rotational component being able to control the pivot-shift phenomenon as revealed by clinical disappearance of the pivot-shift test even before ACLr. However, as ALL repair has no effect on antero-posterior translation which remains evident at navigation, possibly leading to persistent instability, both reconstructions should be recommended in order to better control translation and rotational component of the pivot-shift phenomenon.

The role of ALL in rotational control of the knee has been demonstrated by several robotic cadaveric studies where the effect of the lesion of ACL and ALL were evaluated. Sonnery-Cottet et al (16) reported that ALL is involved in rotational control of the knee at varying degrees of knee flexion and during a simulated pivot-shift in a cadaveric model using a navigation system. Rasmussen et al (15) concluded that ALL is an important lateral knee structure that provides rotational stability of the knee in a cadaveric model using a $6^{\circ}$-degree freedom robotic system and a simulated pivot-shift in ACL deficient knee. Monaco et al. (22) evaluated the effect of a progressive lesion of ACL and
ALL in a cadaveric model and the same navigation system of the present study and they concluded that lesion of the ALL in an ACL deficient knee significantly increases tibial rotation and is correlated to the clinical appearance of an explosive pivot-shift in most cases. Bonazinga et al. (10) evaluated the effect of progressive lesions of ACL and ALL during pivot-shift test. They specifically evaluated acceleration during the pivot shift test. This is a parameter that has been shown to correlate with the clinical grade of pivot shift. They found that while an isolated lesion of the ACL did not significantly affect acceleration of the pivot shift test, an additional injury of the ALL resulted in a significant increase. They postulated that when high grade pivots occur in vivo this may indicate an unrecognised injury to the anterolateral structures of the knee. However, although laboratory studies have played an important role in defining anatomy, their major limitation lies in a difficulty to provide valid extrapolation of biomechanical data from cadaveric specimens (with artificially created injuries and an absence of dynamic muscle forces) to real-world, high functional demand, dynamic clinical scenarios.

In a recent in vivo study (12) prevalence and pattern of lateral injuries were reported in 60 consecutive patients that underwent an acute ACLr (within 7 days after injury). They found that a different type of lesion was observed in $90 \%$ of cases, and only $10 \%$ of these cases showed a Segond fracture detected by X-ray. So they concluded that Segond fracture (Type IV) should be could be considered as the tip of the iceberg of a series of complex injuries more often involving the lateral compartment of the knee and occurring in $90 \%$ of cases in acute, apparently isolated ACL tears. In particular, they found $21 \%$ of type III lesions, involving only the ALL. The aim of this study was to evaluate the effect of an acute repair of the lesions involving only the ALL (Type III lesions) in patients with an acute ACL tear.

To our knowledge, this is the first attempt to describe the in vivo biomechanics effect of the repair of the ALL and our results seems to confirm the importance of this structure in controlling rotational stability of the knee and pivot-shift. In the past, several different extra-articular tenodesis have been considered with the aim to improve rotational stability of the knee. These procedures which are peripheral to the center of rotation of the knee have a better lever arm provided by intra-articular reconstruction for rotatory control (23). The efficacy of this procedure when associated to ACLr, has been demonstrated in long term follow-up studies $(24,25)$ such as in kinematics studies $(22,26-28)$. However, all these procedures are not based on the restoration of anatomic structures, while if an anatomic structure is torn the goal should be to reconstruct its anatomy to as close to native as possible (29,30). For this reason, this paper was focused 
only on patients presenting a complete lesion of the ALL (Type III) (12) to evaluate the effect of a direct repair of this structure.

The major limitation of the present study is that we did not consider the possible spontaneous healing of this kind of lesion making the surgical repair unnecessary. Further studies are needed to obtain a sort of algorithm of treatment including clinical examination with the pivot-shift test, imaging and baseline characteristic of patients (age, sex, sports activity level, etc..) to better understand when an additional procedure to central ACLr and which procedure is needed (laterar extra-articular tenodesis, anatomical ALL reconstruction or acute repair).

There are also some other limitations. First, this is a time 0 study evaluating only the effect on the proposed procedure in a surgical setting, not considering either long-term follow-up clinical results or possible spontaneous healing of the lesion. Secondly, the sequence of the two surgical procedures evaluated was always the same (ALL repair first and ACLr second), but the small sample size didn't allow us to reverse the procedure in a control group. Thirdly, is the limited number of

\section{REFERENCES}

1. Slocum DB, James SL, Larson RL, Singer KM. Clinical test for anterolateral rotary instability of the knee. ClinOrthopRelat Res. 1976; (118):63-9.

2. Tashman S, Collon D, Anderson K., et al. Abnormal rotational knee motion during running after anterior cruciate ligament reconstruction. Am J Sports Med. 2004;32(4):975-83.

3. Ardern CL, Webster KE, Taylor NF, Feller JA. Return to sport following anterior cruciate ligament reconstruction surgery: a systematic review and meta-analysis of the state of play. Br J Sports Med.2011;45(7):596-606.

4. Eriksson E. How good are the results of ACL reconstruction? Knee SurgSportsTraumatolArthrosc. 2011;5(3):137.

5. Musahl V, Griffith C, Irrgang JJ, Hoshino Y, Kuroda R, Lopomo N, Zaffagnini S, Samuelsson K, Karlsson J; PIVOT Study Group. Validation of Quantitative Measures of Rotatory Knee Laxity. Am J Sports Med. 2016 Sep;44(9):2393-8.

6. Lopomo N, Zaffagnini S, Amis AA. Quantifying the pivot shift test: a systematic review. Knee Surg Sports Traumatol Arthrosc. 2013 Apr;21(4):767-83.

7. Claes S, Vereecke E, Maes M, et al. Anatomy of the anterolateral ligament of the knee. J Anat. 2013;223(4):321-8.

8. Helito CP, Demange MK, Bonadio MB, et al. Radiographic landmarks for locating the femoral origin and tibial insertion of the knee anterolateral ligament. Am J Sports Med.2014;42(10): 2356-62.

9. Kennedy MI, Claes S, Fuso FA, et al. The Anterolateral Ligament: An Anatomic, Radiographic, and Biomechanical Analysis. Am SportsMed. 2015;43(7):160615.

10. Bonanzinga T, Signorelli C, Grassi A, Lopomo et al. Kinematics of ACL and anterolateral ligament. Part I: Combinedlesion. Knee Surg Sports TraumatolArthrosc. 2017;25(4):1055-1061. patients, but the type III lesion occurs only in some cases of acute ACL injury (about 20\%). Moreover, we must consider the complexity of an in vivo intra-operative study, which involves navigation and requires a specific informed consent by patients to accept an additional invasive procedure.

The role of the ALL in rotational control of the ACL deficient knee has been clearly demonstrated. Moreover, lesions of the ALL are often associated with acute ACL injuries. Exploration and repair of such lesions at the time of surgery in acute ACLr have a significant effect in reducing rotational component of the pivot-shift as evaluated in vivo by a navigation system, while ACLr has a significant effect in reducing translational component. For this reason, even if isolated ALL repair is able to clinically reduce the pivotshift, ACLr is needed to better control the translational component thus restoring the center of rotation of the knee avoiding residual instability of the knee.

\section{Conflict of Interest}

The authors declare that they have no conflict of interest

11. Noyes FR. Editorial Commentary: Lateral Extra-Articular Reconstructions With Anterior Cruciate Ligament Surgery: Are These Operative Procedures Supported by In Vitro Biomechanical Studies? Arthroscopy. 2016;32(12):2612-2615.

12. Ferretti A, Monaco E, Fabbri M, et al. Prevalence and Classification of Injuries of Anterolateral Complex in Acute Anterior Cruciate Ligament Tears. Arthroscopy. 2017;Jan;33(1):147154.

13. Maeda S, Tsuda E, Yamamoto Y, et al. Quantification of the pivot-shift test using a navigation system with non-invasive surface markers. Knee Surg Sports Traumatol Arthrosc. 2016;24(11):3612-3618.

14. Padulo J, Oliva F, Frizziero A, Maffulli N. Muscles, Ligaments and Tendons Journal - Basic principles and recommendations in clinical and field Science Research: 2018 update. MLTJ 2018; 8(3): $305-307$.

15. Rasmussen MT, Nitri M, Williams BT, et al. An In Vitro Robotic Assessment of the Anterolateral Ligament, Part 1: Secondary Role of the Anterolateral Ligament in the Setting of anAnterior Cruciate Ligament Injury. Am J Sports Med. 2016;44(3):585-92.

16. Sonnery-Cottet B, Lutz C, Daggett M, et al. The Involvement of the Anterolateral Ligament in Rotational Control of the Knee. Am J Sports Med. 2016;44(5):1209-14.

17. Sonnery-Cottet B, Daggett M, Fayard JM, et al. Anterolateral Ligament Expert Group consensus paper on the management of internal rotation and instability of the anterior cruciate ligament - deficient knee. J OrthopTraumatol. 2017;18(2):91-106.

18. Helito CP, Helito PV, Costa HP, et al. MRI evaluation of the anterolateral ligament of the knee: assessment in routine 1.5-T scans. Skeletal Radiol. 2014;43(10):1421-7. 
19. Helito CP, Demange MK, Helito PV, et al. Evaluation of the anterolateral ligament of the knee by means of magnetic resonance examination. Rev Bras Ortop. 2015; 7;50(2):214-9.

20. Bull AM1, Andersen HN, Basso O, Targett J, Amis AA. Incidence and mechanism of the pivot shift. An in vitro study. Clin Orthop Relat Res. 1999 Jun;(363):219-31.

21. Amis AA, Bull AM, Lie DT. Biomechanics of rotational instability and anatomic anterior cruciate ligament reconstruction. OperTechOrthop. 2005;15(1):29-35.

22. Monaco E, Ferretti A, Labianca L, et al. Navigated knee kinematics after cutting of the ACL and its secondary restraint. Knee Surg Sports Traumatol Arthrosc. 2012;20(5):870-7.

23. Slette EL, Mikula JD, Schon JM, Marchetti DC, Kheir MM, Turnbull TL, LaPrade RF. Biomechanical Results of Lateral Extra-articular Tenodesis Procedures of the Knee: A Systematic Review. Arthroscopy. 2016 Dec;32(12):2592-2611.

24. Ferretti A, Monaco E, Ponzo A, et al. Combined Intra-articular and Extra-Articular Reconstruction in Anterior Cruciate Ligament-Deficient Knee: 25 Years Later. Arthroscopy. 2016;32(10):2039-2047.

25. Pernin J, Verdonk P, Si Selmi TA, at al. Long-term followup of 24.5 years after intraarticular anterior cruciate ligament reconstruction with lateral extra-articular augmentation. Am J Sports Med. 2010;38(6):1094-102.

26. Monaco E, Labianca L, Conteduca F, et al. Double bundle or single bundle plus extra articulartenodesis in ACL reconstruction? A CAOS study. Knee Surg Sports TraumatolArthrosc. 2007;15(10):1168-74.

27. Monaco E, Maestri B, Conteduca F, et al. Extra-articular ACL Reconstruction and Pivot Shift: In Vivo Dynamic Evaluation With Navigation. Am J Sports Med. 2014;42(7):16691674.

28. Monaco E, Lanzetti RM, Fabbri M, et al. Anterolateral ligament reconstruction with autologous grafting: A biomechanicalstudy. ClinBiomech (Bristol, Avon). 2017;44:99-103.

29. Musahl V, Rahnemai-Azar AA, Costello J, et al. The Influence of Meniscal and Anterolateral CapsularInjury on Knee Laxity in Patients With Anterior Cruciate Ligament Injuries. Am J Sports Med. 2016; 44(12):3126-3131.

30. Trinchese GF, Oliva F, Maffulli N. Minimally invasive anatomic reconstruction of the anterolateral ligament with ipsilateral gracilis tendon. Muscles Ligaments Tendons J. 2017 Sep $18 ; 7(2): 240-246$. 